\title{
Successful Treatment of Cardiac Failure Due to Cardiomyopathy in Propionic Acidemia by Cardiac Resynchronization Therapy and Hemodialysis in a Young Adult
}

\author{
Masato Kimura1 $^{*}$, Yuji Wakayama², Osamu Sakamoto1, Kengo Kawano', \\ Toshihiro Ohura' ${ }^{1,3}$, Shigeo Kure ${ }^{1}$ \\ ${ }^{1}$ Department of Pediatrics, Tohoku University Graduate School of Medicine, Sendai, Japan \\ ${ }^{2}$ Division of Cardiovascular Medicine, Tohoku University Graduate School of Medicine, Sendai, Japan \\ ${ }^{3}$ Present address: Division of Pediatrics, Sendai City Hospital, Sendai, Japan \\ Email: ${ }^{*}$ mkimura774@med.tohoku.ac.jp
}

Received 3 February 2014; revised 25 February 2014; accepted 4 March 2014

Copyright (C 2014 by authors and Scientific Research Publishing Inc.

This work is licensed under the Creative Commons Attribution International License (CC BY).

http://creativecommons.org/licenses/by/4.0/

c) (i) Open Access

\section{Abstract}

Propionic acidemia is an autosomal recessive disorder that is due to deficiency in the enzyme propionyl-CoA carboxylase. Cardiomyopathy is a well-known phenomenon in propionic acidemia that it may rapidly progress to death. Here we describe a case of propionic acidemia in a 27-yearold man who developed adult-onset secondary dilated cardiomyopathy. In early infancy he was diagnosed with propionic acidemia and was later noted to have mild mental retardation, mild renal failure, and optic nerve atrophy. Although he was in good energy status with a low-protein diet and carnitine supplementation, he was admitted to our university hospital with decompensate heart failure, which resulted in low-output cardiac syndrome with massive mitral regurgitation and left ventricular dyssynchrony. Cardiac resynchronization therapy (CRT) and continuous hemodiafiltration followed by hemodialysis (HD) dramatically improved his clinical status.

\section{Keywords}

Propionic Acidemia; Cardiomyopathy; Cardiac Resynchronization Therapy; Hemodialysis

\section{Introduction}

Propionic acidemia is a relatively rare autosomal recessive disorder characterized by an accumulation of propio-

*Corresponding author.

How to cite this paper: Kimura, M., et al. (2014) Successful Treatment of Cardiac Failure Due to Cardiomyopathy in Propionic Acidemia by Cardiac Resynchronization Therapy and Hemodialysis in a Young Adult. Open Journal of Pediatrics, 4, 79-83. http://dx.doi.org/10.4236/ojped.2014.41011 
nic acid caused by a deficiency in propionyl CoA carboxylase (PCC), an enzyme that catalyzes valine, isoleucine, methionine, threonine, and odd-chain fatty acids in the mitochondrial matrix [1]. PCC deficiency causes an accumulation of propionic acid in the blood and urine, and without appropriate treatment, can lead to metabolic acidosis, lethargy, respiratory distress, and death. Recent advances have enabled patients with acute presentation to live beyond the neonatal period. As patients age, however, the natural progression of propionic acidemia gives rise to intellectual difficulties and increases the risks for a number of complications, including chronic kidney disease and neurological, cardiac, and gastrointestinal disorders [2]-[4]. The initial onset of cardiomyopathy typically occurs later, during school age or in adulthood, but despite its later onset, it is a serious complication as it can rapidly progress to death [5].

Cardiac resynchronization therapy (CRT) is an established adjunct to optimal pharmacological therapy in eligible patients with severe heart failure, but its role in the treatment of heart failure due to secondary cardiomyopathy has not been clarified [6] [7]. Here we describe the case of a young adult with propionic acidemia complicated by severe heart failure and renal dysfunction who was treated successfully by combination therapy CRT and HD. This combined approach may be a viable option for patients with metabolic disorders such as propionic acidemia complicated advanced secondary cardiomyopathy and renal failure.

\section{Case Report}

A 27-year-old Japanese man with propionic acidemia presented to our university hospital with shortness of breath and nocturnal dyspnea. He was the third child of a nonconsanguineous marriage [8]. His older sister had died of unknown causes during the neonatal period. He was born by normal delivery with a weight of $3200 \mathrm{~g}$. Shortly after birth, he exhibited poor feeding, lethargy, and respiratory distress and underwent peritoneal dialysis together with other standard measures to control metabolic disorder. Diagnosis of propionic acidemia was confirmed by plasma and urinary amino acid analysis. Mutation analysis revealed homozygosity for p.Thr428Ile in the PCCB gene, which codes the beta subunit of PCC. He grew normally on a low-protein diet supplemented with L-carnitine, but had mild mental retardation. At age 15 years, he collapsed and was diagnosed with epilepsy on the basis of abnormal electroencephalography findings. Zonisamide was administered to control his convulsive seizures and he managed to work part time until losing his vision due to optic atrophy at age 22. Cardiomegaly became apparent at age 23 , with no family history of cardiomyopathy.

On admission, physical examination revealed height of $1.57 \mathrm{~m}, 56.5 \mathrm{~kg}$ weight, clear consciousness, tachypnea, alternating pulse, and clammy skin at the extremities. A systolic murmur (Levine II/VI) with a gallop rhythm was audible at the apex. Chest radiography showed cardiomegaly and bilateral pulmonary congestion with a cardiothoracic ratio of 60\% (Figure 1(a)). Electrocardiography demonstrated sinus tachycardia with normal QRS (100 ms in V6) and QT (425 ms in V5) intervals. Echocardiography showed notable global left ventricular dysfunction, characterized by a left ventricular ejection fraction of $15.6 \%$ and a markedly dilated left ventricular cavity. Doppler echocardiography revealed severe mitral regurgitation (Figure 1(c)). The results of blood gas analysis were as follows: $\mathrm{pH}, 7.409$; $\mathrm{PCO}_{2}, 32.5 \mathrm{mmHg} ; \mathrm{PO}_{2}, 48.1 \mathrm{mmHg}$; bicarbonate, $18.5 \mathrm{mmol} / 1$; $\mathrm{BE},-9.5$; glucose, $125 \mathrm{mg} / \mathrm{dl}$; and lactate, $14.5 \mathrm{mg} / \mathrm{dl}$. Blood ammonia was within the normal range, serum creatinine was mildly elevated $(1.8 \mathrm{mg} / \mathrm{dl})$, and brain natriuretic peptide was notably elevated $(2000 \mathrm{pg} / \mathrm{ml})$. Diuretics, carperitide, catecholamines, and nasal continuous positive airway pressure did not improve his respiratory distress or heart failure. He was intubated and mechanically ventilated due to severe respiratory distress with pulmonary congestion. He developed oliguria and his serum creatinine level was elevated to 3.4 (mg/dl). Continuous hemofiltration was initiated to treat low cardiac output and pulmonary congestion and significantly improved his clinical status and pulmonary congestion. However, he needed regular hemodialysis (HD) to remove the excess preload. He also experienced ventricular fibrillation and required resuscitation on a general ward, but showed no precursory change. Implantation of a CRT defibrillator system (Medtronic Protecta ${ }^{\mathrm{TM}}$ XT, Medtronic Inc, Minneapolis, USA) and periodic HD gradually improved his cardiac function. He was discharged with the same neurological status admission with a cardiothoracic ratio of 50\% (Figure 1(b)) and has not had any cardiac events for 3 years with periodic HD three times a week.

\section{Discussion}

Cardiomyopathy as a complication of organic acidemia, particularly propionic acidemia, was first described in 1993 by Massoud et al. in 6 of 19 pediatric patients with propionic academia [2] and is now a well-established 




Figure 1. Chest X-rays and echocardiographs on admission (a, b) and at 7 months after introducing the cardiac resynchronization therapy defibrillator (CRTD) system (c, d). (a) Cardiomegaly (cardiothoracic ratio, 60\%) with pulmonary congestion. (b) Apical four-chamber images of severe mitral insufficiency showing mitral annular dilatation (left panel) and severe mitral regurgitation on color flow Doppler (right panel, arrow). (c) cardiothoracic ratio of $50 \%$ with no pulmonary congestion. (d) Apical four-chamber images of mild mitral regurgitation on color flow Doppler (right panel, arrowhead) showing a marked reduction in mitral regurgitation after introducing the CRTD system and hemodialysis.

phenomenon [Pena, Burton 2012]. Among their 6 patients, 3 died of cardiomyopathy and the other 3 fully recovered. A recent survey of propionic acidemia complications found that cardiomyopathy occurred in 10 of 54 (19\%) patients with propionic acidemia, aged 2 - 33 years old [3]. Importantly, propionic acidemia was listed as the cause of death in $70 \%$ of the deceased patients, a finding indicative of significant morbidity and mortality in this population.

To date, the cause of cardiomyopathy has not been elucidated, and although carnitine deficiency has been observed in patients with cardiomyopathy, carnitine therapy has not shown any beneficial effects in patients with propionic acidemia-related cardiomyopathy [2]. Mardach et al. reported very low levels of cardiac muscle carnitine despite normal plasma carnitine levels, and subsequently proposed that depletion of cardiac muscle carnitine is a precursor of cardiomyopathy [9]. However, as cardiac myocyte findings were limited, further research is needed to clarify any potential relationship. Mitochondrial electron transport chain dysfunction and secondary respiratory chain deficiency followed by an accumulation of toxic metabolites in tissues from patients with propionic acidemia are potential etiologic factors in the development of cardiomyopathy and other complications [10]. While liver transplantation has been shown to ameliorate cardiomyopathy, the absence of a correlation between their metabolic state and this heart complication suggests that different mechanisms may be involved [11]. Biventricular stimulation (i.e., CRT) has been shown to improve cardiac function, reduce the frequency of hospitalization for heart failure, and enhance the quality of life in many patients with severe left ventricular systolic dysfunction and intraventricular conduction disease [6] [7]. However, it remains controversial as to whether CRT benefits patients with heart failure due to secondary cardiomyopathy such as metabolic and/or degenerative disease. In the present case, the introduction of a CRT device significantly improved the ejection fraction, magnitude of mitral regurgitation, and left ventricular dyssynchrony (Figure 2). It is possible that combination therapy, preload reduction, the removal of toxic substances by HD, and the application of CRT to assist cardiac contractions and rectify cardiac dyssynchrony have a synergistic effect.

In summary, combination therapy with CRT and HD dramatically improved the condition of a young man with propionic acidemia complicated by severe left heart failure and renal dysfunction. This combined approach 




Figure 2. Speckle-tracking images showing dyssynchrony of peak segmental radial strain on admission (a) and at 7 months after introducing the CRTD system (b). Dyssynchrony was assessed using proprietary software (Toshiba Medical Systems Corporation, Tokyo, Japan). a: Dyssynchrony is shown as the time difference (arrow) between time to peak strain in the anterior (yellow outline) and lateral (blue outline) walls and time to peak strain in the inferior wall (purple outline) and septum (pink outline). Data in the table indicate marked cardiomegaly with an end diastolic volume of $426 \mathrm{ml}$. (b) CTRD placement resulted in improved synchrony of segmental shortening accompanied by a global systolic function left ventricular ejection fraction of $34.8 \%$ (compared with $15.6 \%$ in panel a).

may be a viable option for individuals with metabolic diseases such as propionic acidemia complicated by advanced secondary cardiomyopathy with renal dysfunction.

\section{Acknowledgements}

We thank Koji Fukuda, the consultant cardiologist of Division of Cardiovascular Medicine, Tohoku University Graduate School of Medicine. We would also thank the institutions where the work was performed, namely Department of Pediatrics, Tohoku University Graduate School of Medicine, Sendai, Miyagi, Japan

\section{References}

[1] Scholl-Burgi, S., Sass, J.O., Zschocke, J. and Karall, D. (2012) Amino Acid Metabolism in Patients with Propionic Acidaemia. Journal of Inherited Metabolic Disease, 35, 65-70. http://dx.doi.org/10.1007/s10545-010-9245-9

[2] Massoud, A.F. and Leonard, J.V. (1993) Cardiomyopathy in Propionic Acidaemia. European Journal of Pediatrics, 
152, 441-445. http://dx.doi.org/10.1007/BF01955907

[3] Pena, L. and Burton, B.K. (2012) Survey of Health Status and Complications among Propionic Acidemia Patients. American Journal of Medical Genetics Part A, 158A, 1641-1646. http://dx.doi.org/10.1002/ajmg.a.35387

[4] Vernon, H.J., Bagnasco, S., Hamosh, A. and Sperati, C.J. (2013) Chronic Kidney Disease in an Adult with Propionic Acidemia. Journal of Inherited Metabolic Disease Reports, 12, 5-10. http://dx.doi.org/10.1007/8904_2013_237

[5] Pena, L., Franks, J., Chapman, K.A., Gropman, A., Mew, N.A., Chakrapani, A., Island, E., Matern, D., Smith, B., Stagni, K., Sutton, V.R., Ueda, K., Venditti, C., Enns, G.M. and Summar, M.L. (2012) Natural History of Propionic Acidemia. Molecular Genetics and Metabolism, 105, 5-9. http://dx.doi.org/10.1016/j.ymgme.2011.09.022

[6] Chen, S., Yin, Y. and Krucoff, M.W. (2012) Effect of Cardiac Resynchronization Therapy and Implantable Cardioverter Defibrillator on Quality of Life in Patients with Heart Failure: A Meta-Analysis. Europace, 14, 1602-1607. http://dx.doi.org/10.1093/europace/eus168

[7] Holzmeister, J. and Leclercq, C. (2011) Implantable Cardioverter Defibrillators and Cardiac Resynchronisation Therapy. Lancet, 378, 722-730. http://dx.doi.org/10.1016/S0140-6736(11)61228-2

[8] Komatsuzaki, S., Sakamoto, O., Fuse, N., Uematsu, M., Matsubara, Y. and Oura, T. (2012) A Young Male Patient with Progressive Subcortical Lesions and Optic Nerve Atrophy. Neurology, 79, e63-68. http://dx.doi.org/10.1212/WNL.0b013e3182648bb6

[9] Mardach, R., Verity, M.A. and Cederbaum, S.D. (2005) Clinical, Pathological, and Biochemical Studies in a Patient with Propionic Acidemia and Fatal Cardiomyopathy. Molecular Genetics and Metabolism, 85, 286-290. http://dx.doi.org/10.1016/j.ymgme.2005.04.004

[10] de Keyzer, Y., Valayannopoulos, V., Benoist, J.F., et al. (2009) Multiple OXPHOS Deficiency in the Liver, Kidney, Heart, and Skeletal Muscle of Patients with Methylmalonic Aciduria and Propionic Aciduria. Pediatric Research, 66, 91-95. http://dx.doi.org/10.1016/j.ymgme.2005.04.004

[11] Romano, S., Valayannopoulos, V., Touati, G., et al. (2010) Cardiomyopathies in Propionic Aciduria Are Reversible after Liver Transplantation. Journal of Pediatrics, 156, 128-134. http://dx.doi.org/10.1016/j.jpeds.2009.07.002 